\title{
EPJ
}

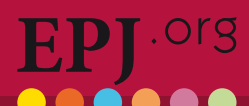

000000

Eur. Phys. J. Plus (2012) 127: 27

DOI 10.1140/epjp/i2012-12027-9

\section{Absolute gravity acceleration measurement in atomic sensor laboratories}

M. de Angelis, F. Greco, A. Pistorio, N. Poli, M. Prevedelli, G. Saccorotti, F. Sorrentino and G.M. Tino 


\title{
Absolute gravity acceleration measurement in atomic sensor laboratories
}

\author{
M. de Angelis ${ }^{\mathrm{a}, \mathrm{b}}$, F. Greco ${ }^{\mathrm{c}}$, A. Pistorio $^{\mathrm{c}, \mathrm{d}}$, N. Poli, M. Prevedellie, G. Saccorottif ${ }^{\mathrm{f}}$, F. Sorrentino, and G.M. Tino \\ Dipartimento di Fisica e Astronomia and LENS, Università di Firenze - INFN Sezione di Firenze \\ via Sansone 1 - Polo Scientifico, I-50019 Sesto Fiorentino, Firenze, Italy
}

Received: 5 September 2011 / Revised: 25 January 2012

Published online: 6 March 2012 - (c) Società Italiana di Fisica / Springer-Verlag 2012

\begin{abstract}
This paper reports the results from the accurate measurement of the acceleration of gravity $g$ taken at two separate premises in the Polo Scientifico of the Florence University (Italy). In these laboratories, two separate experiments aiming at measuring the Newtonian constant and testing the measurement of forces with high spatial resolution are in progress. Both experiments require an independent knowledge on the local value of $g$. Gravity measurements were conducted using an FG5 absolute gravimeter, and accompanied by seismic recordings for evaluating the noise condition at the site. The absolute accelerations of gravity at the two laboratories are $(980492160.6 \pm 4.0) \mu \mathrm{Gal}$ and $(980492048.3 \pm 3.0) \mu \mathrm{Gal}$ for the European Laboratory for Non-Linear Spectroscopy (LENS) and Dipartimento di Fisica e Astronomia, respectively. Other than for the two referenced experiments, the data here presented will serve as a benchmark for any future study requiring an accurate knowledge of the absolute value of the acceleration of gravity in the study region.
\end{abstract}

\section{Introduction}

Methods based on quantum interference of ultracold atoms, have recently led to the development of new techniques for the measurement of inertial forces, with important applications both in fundamental physics and applied research. The remarkable stability and accuracy that atom interferometers have reached for acceleration measurements can play a crucial role for gravimetry. Atom interferometry is used for precise measurements of gravity acceleration, Earth's gravity gradient and rotations. Accelerometers based on atom interferometry have been developed for many practical applications including metrology, geodesy, geophysics, engineering prospecting and inertial navigation (see [1] and references therein).

Over the past few years two separate experiments, one for measuring the Newtonian constant $G$ and one for testing the gravity on micrometric spatial resolution, are under development at the physics laboratories of the Florence University. The experiment for the Newtonian constant measurement is based on an atomic gradiometer that detects the differential acceleration induced by very well-known source masses $[2,3]$. In this apparatus, two clouds of lasercooled rubidium atoms are launched in a fountain configuration and simultaneously interrogated by a Raman-pulse interferometry sequence. From the differential acceleration measurement and from the knowledge of the added mass distribution, it is possible to determine the value of the Newtonian constant. A reasonably accurate value of $g$ is also needed, because it enters the calculation of atomic trajectories in the $G$ measurement. The system has recently been upgraded and its stability evaluated [4]. The signal-to-noise ratio and the long-term stability of the gravity gradiometer demonstrated interesting perspectives for pushing the $G$ measurement uncertainty below the $1 \times 10^{-4}$ level, corresponding to an uncertainty of $\simeq 2 \times 10^{-11} \mathrm{~g}$ of differential gravity acceleration. Due to the differential nature of the measurement, several noise sources are rejected as common noise and there is no need, for instance, to insulate

\footnotetext{
a Present address: Istituto di Fisica Applicata "Nello Carrara" CNR, Via Madonna del Piano 10, I-50019 Sesto Fiorentino, Italy.

b e-mail: marella.deangelis@fi.infn.it

c Present address: Istituto Nazionale di Geofisica e Vulcanologia, Sezione di Catania, P.zza Roma 2, I-95125 Catania, Italy.

d Also at: Dipartimento di Ingegneria Elettrica, Elettronica e Informatica - Università degli Studi di Catania, Italy.

e Present address: Dipartimento di Fisica, Università di Bologna, Via Irnerio 46, I-40127 Bologna, Italy.

f Present address: Istituto Nazionale di Geofisica e Vulcanologia, Sezione di Pisa, Via della Faggiola 32 - I-56126 Pisa, Italy.
} 
from the seismic noise. Nevertheless with an accurate value for $g$ it is possible to check our instrument against various systematic effects.

The experiment for gravity measurements tests on micrometric scale is based on optically trapped strontium atoms. The small size and high sensitivity of the atomic probe allow a model-independent acceleration measurement at distances of a few $\mu \mathrm{m}$ from a source mass, giving direct access to a poorly tested range of the Newtonian law [5]. The experiment is also of relevance for the recent interpretation of related experiments as tests of gravitational redshift [6, 7]. In the experiment, laser-cooled strontium atoms are trapped in a vertical optical lattice and the combination of the periodic optical potential and the linear gravitational potential gives rise to Bloch oscillations. From the measured Bloch frequency the gravity acceleration along the optical lattice is estimated. In this experiment the comparison with an independent measurement of the absolute gravity acceleration can check the long-term stability of the atom probe for the investigation of forces.

In this paper, we report in detail the measurement of gravity acceleration with an independent instrument. Considering that the uncertainty of atom sensors in the measurement of $g$ is limited to a level of $10^{-7}$ for the strontium Bloch oscillations gravimeter and to $10^{-6}$ for the rubidium Raman gravity gradiometer, an instrument with an uncertainty of 1 part in $10^{7}$ or better is suitable for the comparison in the sites where the atom sensor gravity measurements are running.

The absolute acceleration of gravity $g$ has been measured using the Microg-LaCoste FG5\#238 absolute gravimeter in the laboratories of the two experiments at the Polo Scientifico of Florence University in Sesto Fiorentino. As far as we know, the closest and most recent measurement of the acceleration of gravity is the measurement realized beneath the Italian Zero Order Gravity Net [8]. The measurement was performed at Palazzo al Piano (about $30 \mathrm{~km}$ from our location) in 1989 where a $g$ value at ground of $980391580(8) \mu \mathrm{Gal}$ was found, and it was performed using the absolute gravimeter of the IMGC (now INRIM) in Turin (see [9] and references therein). Of course, the value of absolute $g$ could be derived from the one taken in [8] to our location using a relative spring gravimeter, but the main limit is that there is no information on the stability of the measurements in Palazzo al Piano since measurements have not been repeated [10] on this site. The poor reliability of the operation persuaded us to measure absolute $g$ directly in our laboratories.

In this paper, we discuss the measurements of the absolute acceleration of gravity taken in Firenze in the period October 4th-6th, 2009. In sect. 2 we describe the atom sensor experiments. In sect. 3 we present the general description of the site: sect. 3.1 outlines the geological setting and sect. 3.2 is dedicated to the study of the seismic noise at the measurement locations. In sect. 4 data processing and the corrections to the measurements are described, and sect. 5 is dedicated to the conclusions.

\section{The atomic sensors experiments}

The experiment for the Newtonian constant measurement is based on an atomic gradiometer that detects the differential acceleration induced by very well-known source masses [2-4]. The atomic gradiometer is a matter wave interferometer where two clouds of laser-cooled ${ }^{8} 7 \mathrm{Rb}$ atoms, separated by a distance $D \simeq 30 \mathrm{~cm}$ are used for a simultaneous measurement of local gravity with respect to the common reference frame identified by the wave fronts of the laser beam used for the atom interference interrogation. The instrument directly detects the differential acceleration between the two clouds and thus the common mode acceleration noise induced on the wave fronts by acoustic and seismic vibrations is rejected. The instrument is an excellent gradiometer since the gravity gradient in the vertical direction is simply the ratio between the differential acceleration and the distance $D$ and it can be determined with a statistical error essentially equal to $\Delta D / D$ (with $\Delta D$ the uncertainty on the measure of $D$ ) which is lower than $0.1 \%$. From the differential acceleration and from the knowledge of the added mass distribution, it is possible to determine the value of the Newtonian constant if a reasonably accurate value of each trajectory apogees is also known. Since the position of the apogees is determined with time-of-flight technique with respect to a reference position in the experiment set-up, it is required to know the value of $g$ within 1 part in $10^{5}$. The reference absolute gravity acceleration value must be set within this uncertainty or better.

Since each atom cloud can be operated also as a gravimeter, $g$ can be obtained by the atom interferometer itself and indeed state-of-the-art instruments based on atomic interference have been built [11]. Due to the differential nature of this instrument, however, no special care has been taken in insulating the experiment from seismic noise; this means that when the instrument is working as a gravimeter, its statistical error is limited, after 3 minutes of integration, to a sensitivity $\Delta g / g=5 \times 10^{-7}$. In this case an independent measurement of the gravity acceleration in the laboratory, with an uncertainty of comparable level or better, can be a good test of the control on the systematic errors of the atom sensor.

In the experiment based on optically trapped strontium atoms, the confinement of ultracold strontium atoms in optical lattices provides clean model systems to tests of gravity at micrometer scale. The small size and high sensitivity of the atomic probe allow a model-independent measurement at distances of a few $\mu \mathrm{m}$ from the source mass, like in $[12$, 13]. In the experiment laser-cooled strontium atoms are trapped in a 1-dimensional vertical optical lattice and the combination of the periodic optical potential and the linear gravitational potential gives rise to Bloch oscillations. 

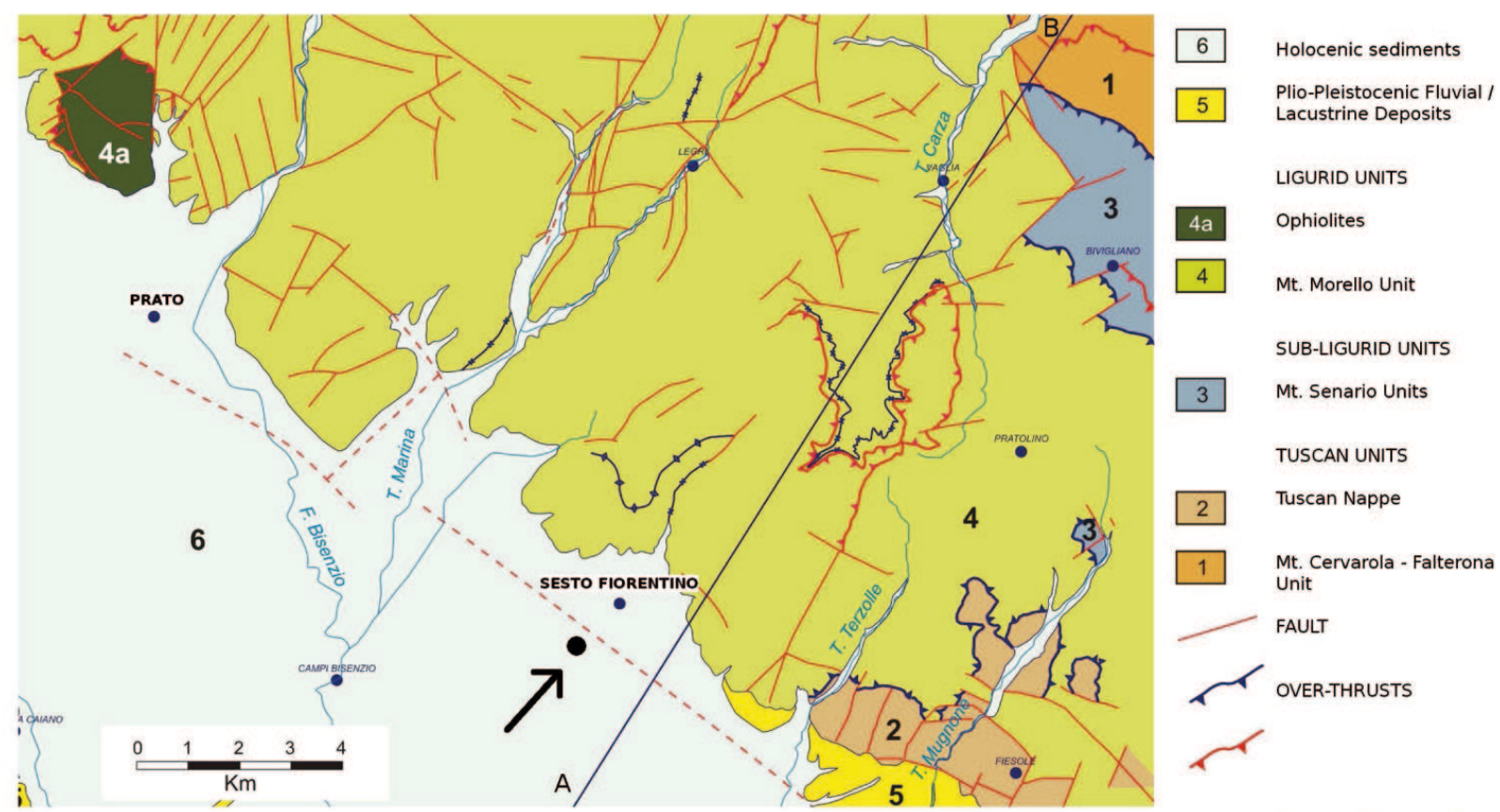

Plio-Pleistocenic Fluvial

LIGURID UNITS

Ophiolites

Mt. Morello Unit

SUB-LIGURID UNITS

Mt. Senario Units

TUSCAN UNITS

Tuscan Nappe

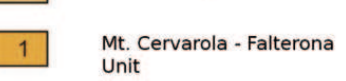

Unit
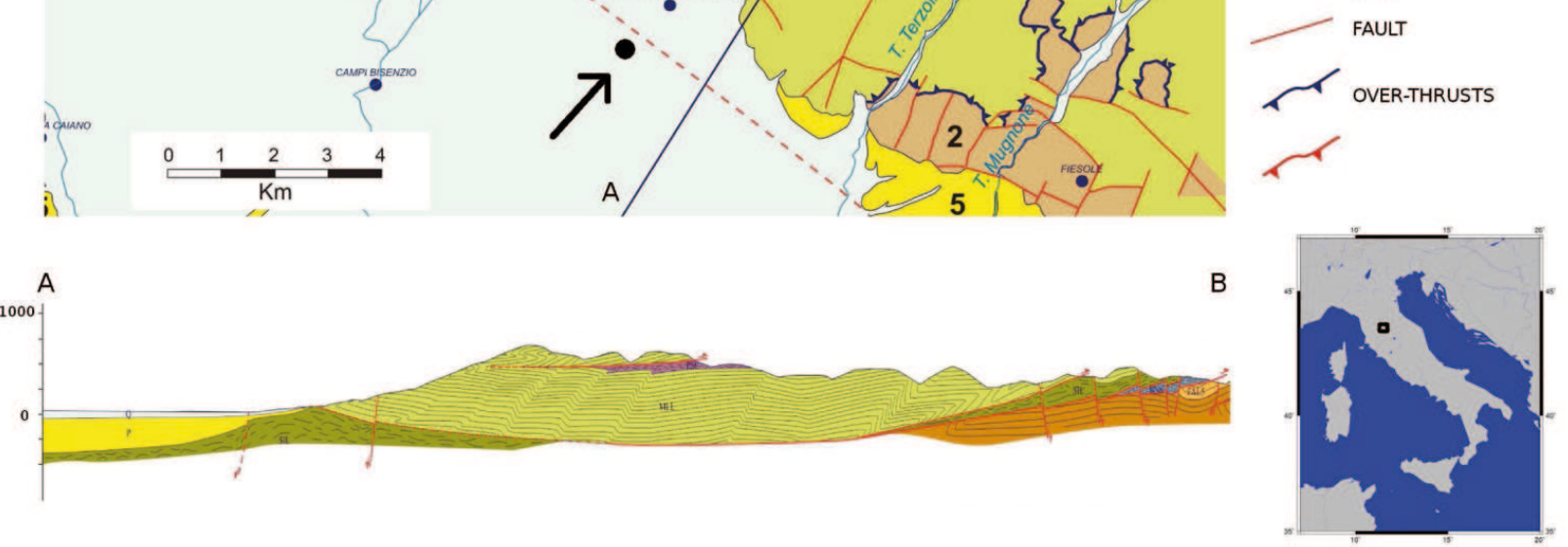

Fig. 1. (Top) Sketch map of the study area, outlining its main geological and structural features. The arrow marks the location of the measurement site. (Bottom) The geological section through points A-B in the map. The inset at the bottom right shows location of the study area with respect to Italy. The map is modified from Sheet 263 of the Geological Cartography series of the Regione Toscana, available at http://www.regione.toscana.it.

From the measured Bloch frequency $\nu_{B}$ the gravity acceleration along the optical lattice is estimated with a sensitivity of $\Delta g / g \simeq 10^{-7}$ after 30 minutes of integration. In this experiment the performance in measuring $g$ is an index of the stability of the atom probe for the investigation of forces at small spatial scales and is of interest as a test of the gravitational redshift.

\section{The site}

The buildings of the Polo Scientifico of the Florence University in Sesto Fiorentino have been constructed recently, and became fully operational by late 2000. The specific sites where the FG5 instrument has been placed are in room 67 (Rb lab) building 3 (Department of Physics and Astronomy) at WGS84 coordinates 43.81918(1) ${ }^{\circ}$ N11.19291(1) ${ }^{\circ}$ E, and in room 44 (Sr lab) in building 4 at WGS84 coordinates $43.81902(1)^{\circ} \mathrm{N} 11.19303(1)^{\circ}$ E. Both laboratories are at the ground floor, at an elevation of about $40 \mathrm{~m}$ above sea level (data from the Gauss-Boaga Fuso 1 map of Regione Toscana, 1:2000 scale). The concrete floor where the instrument is installed is on the basements of the buildings and it is covered by solid tile. In the $\mathrm{Rb}$ lab, air conditioning maintains the temperature at $(20.0 \pm 0.1)^{\circ} \mathrm{C}$, while temperature stability in the $\mathrm{Sr}$ lab is not better than $\pm 3^{\circ} \mathrm{C}$.

\subsection{Geological setting}

The site is located close to the NW border of the middle Valdarno basin, hereinafter referred to as the Firenze-PratoPistoia basin. This is one of the tectonic basins which developed since the Neogene in the Tyrrhenian side of the Apennines thrust and fold belt, striking parallel to the main chain axis (fig. 1). The genesis of these depressions is related to an extensional tectonic regime developed since Upper Tortonian age (11-7 My before present) and due to the 


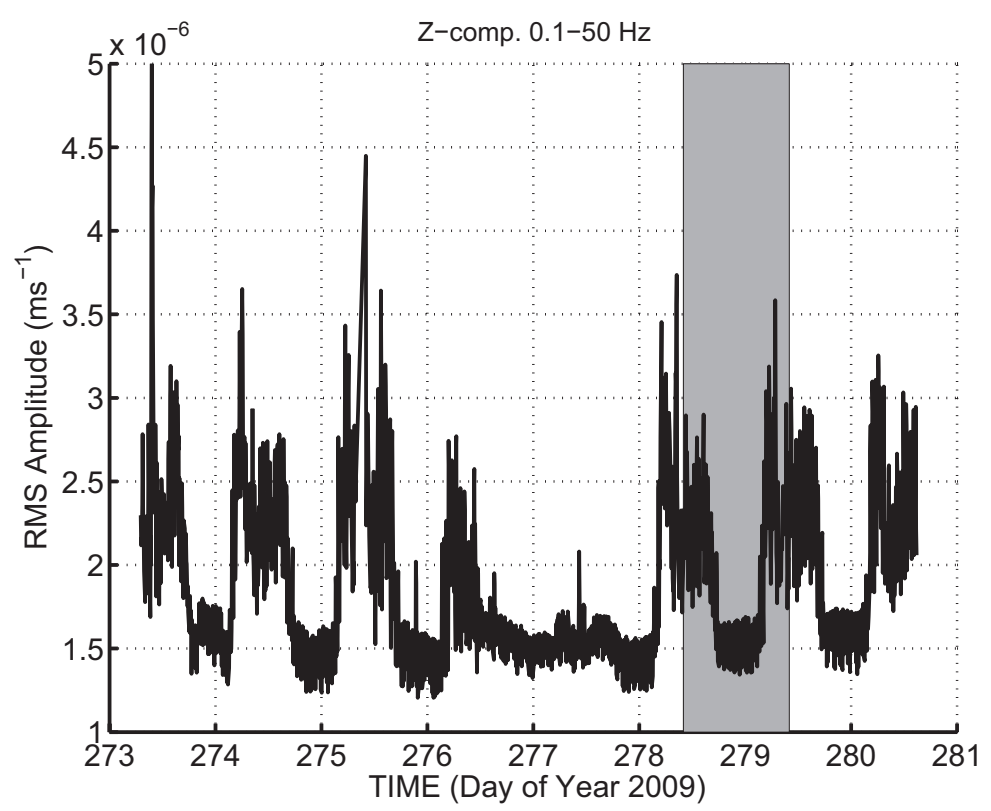

Fig. 2. RMS amplitude of the seismic noise at the measuring site from September 30th, 2009 to October 7th, 2009. Data represent the standard deviation of the $0.1-50 \mathrm{~Hz}$ vertical component of ground velocity computed over $10 \mathrm{~min}$ long time windows.

opening of the Tyrrhenian Sea (see [14] and references therein). The Firenze-Prato-Pistoia basin extends in a NW-SE direction with a roughly rectangular shape and is delimited by Neogene-Quaternary faults, the most important of which is oriented NW-SE bounding the northeastern margin of the basin.

The substratum of the basin is mainly formed by rocks pertaining to the Ligurian Units s.l. (shales, calcareousquarzitic sandstone, calcareous turbidites and marly limestones) that outcrop NE of the basin (Monte Morello-Calvana Ridge) and tectonically overlie the turbiditic formations of the Tuscan Unit (Macigno sandstone). The maximum thickness of the lacustrine deposits in the middle of the basin extends up to $500 \mathrm{~m}$. However, the depth of the bedrock within the basin varies significantly, as a consequence of several NE-SW trending faults which subdivide the bottom of the basin in a block-like structure.

Below the study area, the thickness of the sedimentary cover has been estimated on the order of $450 \mathrm{~m}$ [15]. Four main sedimentary phases are recognised (depths are referred to sea level): from about $-400 \mathrm{~m}$ to $-20 \mathrm{~m}$ : fluviolacustrine succession, constituted by sands, pebbles and clays whose age is generally considered to be Upper PlioceneLower Pleistocene ( $\simeq 2.5 \mathrm{My}$ before present); from $-20 \mathrm{~m}$ to $-5 \mathrm{~m}$ : pebbles, gravels and silt of fluvial fans resulting from erosion of the Monte Morello-Calvana ridge; from $-5 \mathrm{~m}$ to $+10 \mathrm{~m}$ : lacustrine clays and gravels; from $+10 \mathrm{~m}$ to surface: Fluvial clays and gravels.

The static level of the water table varies between $0.5 \mathrm{~m}$ and $2 \mathrm{~m}$ below the surface, as also visible at the numerous drainage channels present in the area [16].

From both direct and indirect measurements, shear wave velocities have been determined to vary between 200 $300 \mathrm{~m} / \mathrm{s}$ at the surface and $600-800 \mathrm{~m} / \mathrm{s}$ at $40 \mathrm{~m}$ depth.

\subsection{Seismic noise}

The site is located within an industrial area, and close to the A11 Highway, the Airport and a major construction site. The intense ground vibrations of both anthropic and natural origin cause an acceleration noise which may induce a significant drop-to-drop scatter of the gravity observations.

In order to quantify the noise conditions at the measuring site, we thus conducted a microseismic survey during an 8-day-long period encompassing the gravity measurements. For these measurements, two Nanometrics Trillium 120P seismometers (http://www.nanometrics.ca) have been used, whose response function is flat over the $120 \mathrm{~s}-0.02 \mathrm{~s}$ period range. Acquisition was performed using two 24 bit Reftek 130 portable recorders, with a digitising rate of 125/samples/second/channel, independently synchronised to the UTC time base via GPS receivers.

Data presented hereinafter are from station A667, which was operated at Rb lab throughout the duration of the microseismic survey. Figure 2 shows the time series of noise amplitude obtained from the standard deviation of consecutive, $600 \mathrm{~s}$ long windows of signal band pass filtered over the $0.1-50 \mathrm{~Hz}$ frequency band using a 2 pole, 0 phase shift Butterworth filter. 


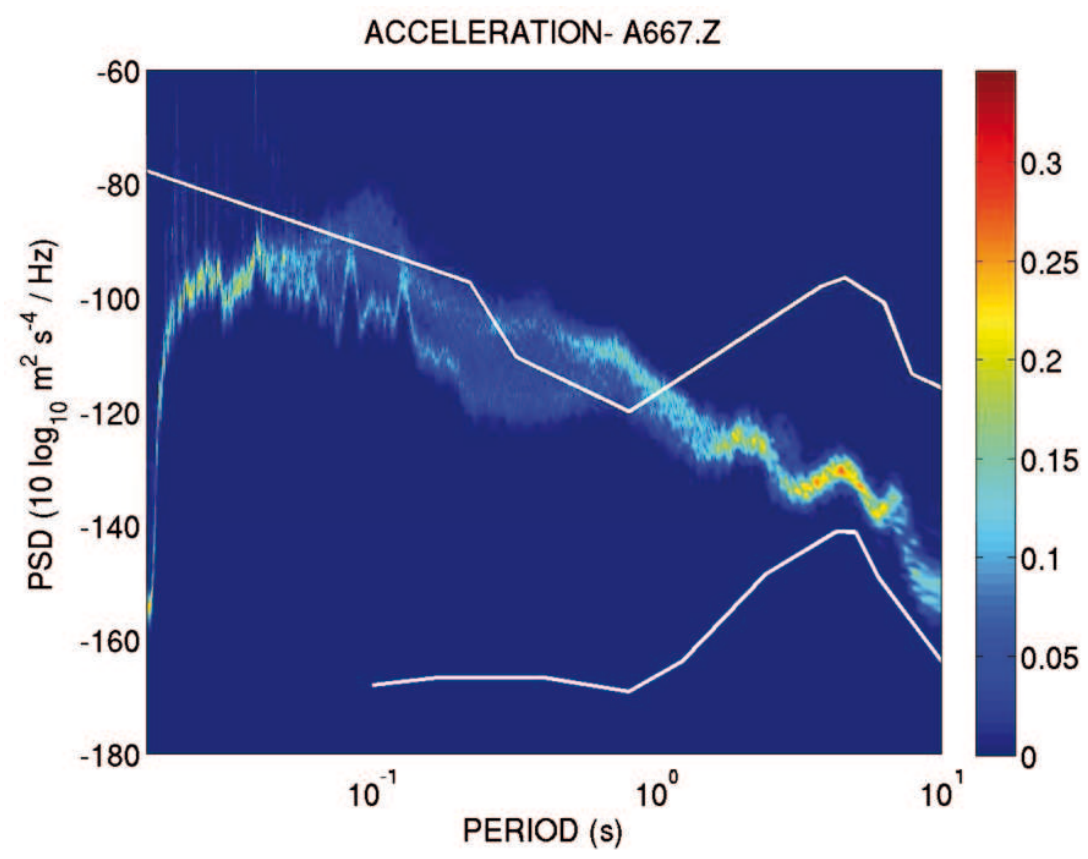

Fig. 3. Probability density function of the vertical-component noise amplitude for a $24 \mathrm{~h}$ long time interval encompassing the gravity measurements. The distribution is obtained by binning at $1 \mathrm{~dB}$ interval the spectral power measured at consecutive discrete Fourier frequencies which, in our case, are spaced by $0.0083 \mathrm{~Hz}$. White lines are the Earth's High- and Low-Noise Models (see [19]).

Seismic noise exhibits a typical weekly and daily pattern, such as the $8 \mathrm{~h}$ workday, due to the intense human activities conducted both inside and in proximity of the laboratories. Ground vibrations at day time are $2.5-3$ times larger than those observed during the night.

Figure 3 illustrates the probability density function (PDF) [17] of acceleration power spectral densities during the $24 \mathrm{~h}$ long period of gravity measurements. The PDF is representative of 130 spectral estimates obtained via Welch's method [18] applied to 10 non-overlapping, $120 \mathrm{~s}$ long windows of noise. Individual spectral estimates have been stabilised using a $0.1 \mathrm{~Hz}$ wide smoothing window. For reference, these data are compared to Peterson's (1993) Lowand High-Noise Model curves [19]. At periods between $5 \mathrm{~s}$ and $10 \mathrm{~s}$, the noise PDFs are very narrow, and their peaks are rather close to the Low-Noise Model. This is not surprising, considering that the main noise source over this particular period range is marine microseismic activity, and the test site is located about $80 \mathrm{~km}$ far from the coast.

At shorter periods (0.05-1 s, corresponding to the 1-20 Hz frequency band), the PDF becomes wider, and encompass the High-Noise Model. The spreading of amplitude distributions over this period range is likely related to the day-night variation of vibrations, thus suggesting a dominance of anthropic sources. At periods shorter than $0.05 \mathrm{~s}$ (frequencies above $20 \mathrm{~Hz}$ ), several narrow spectral peaks indicate the action of non-stationary, monochromatic vibrations from nearby sources, such as the air-conditioning system.

Seismic noise is not a problem for the FG5 instrument after measuring for $12-24$ hours and considering that the reference mirror of the instruments is isolated from vibrations with a "super-spring". This may not be the case for the atom interferometer sensors (see [20]), that are operating in the same site, and moreover if the seismic noise of the site is higher than the High-Noise Model reference curves [19]. In fact, the site is located within an industrial area and the acoustic noise may become an impending point for the measuring performances of the atom sensors. We have compared rms of the measured $g$ value taken with FG5 against the rms of the vertical acceleration of ground taken with the seismometer (see fig. 4) showing correlation between the two.

\section{The absolute gravity acceleration instrument and measurements}

\subsection{The absolute gravimeter}

The Microg-LaCoste FG5\#238 ballistic absolute gravimeter [21] is a high-precision instrument that measures the free fall acceleration of a repeatedly dropped corner cube reflector. This test mass is contained in a cofalling servo-controlled motor-driven drag-free chamber and falls over $20 \mathrm{~cm}$ in $0.2 \mathrm{~s}$ inside a vacuum chamber. A laser interferometer is used to determine the position of the test mass as a function of time during its free fall. This interferometer is a modified 

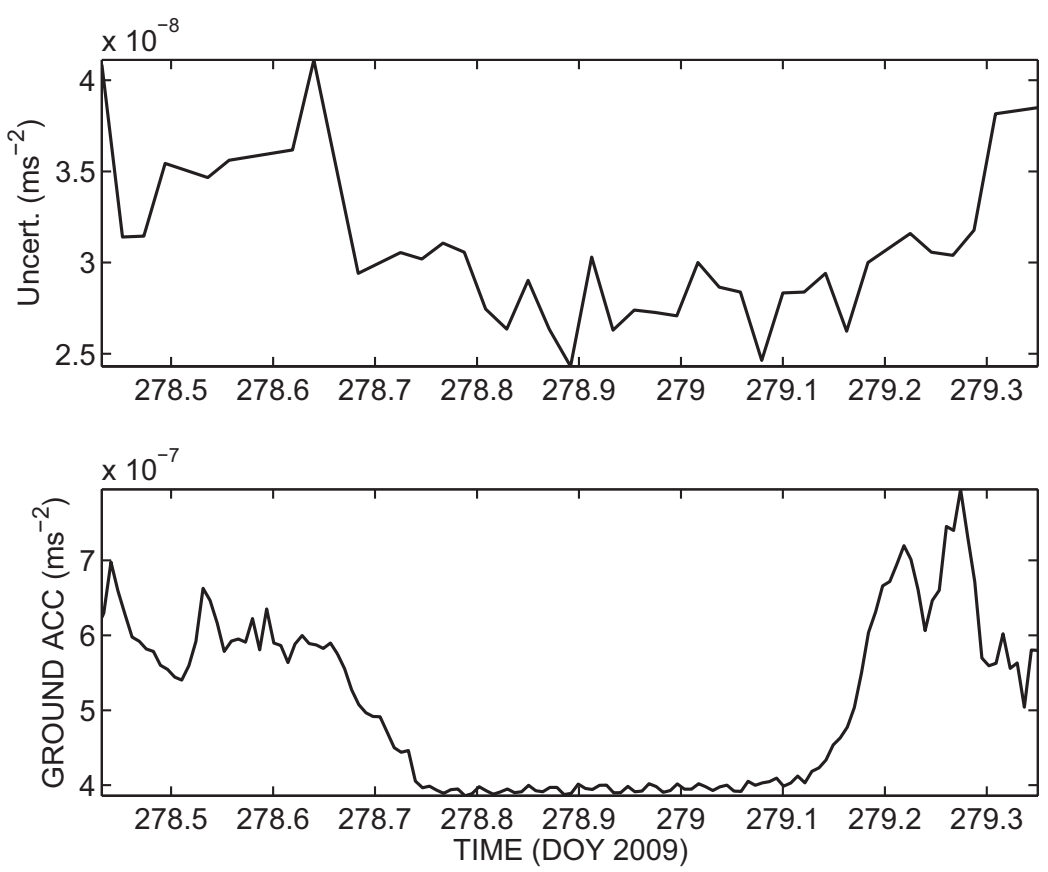

Fig. 4. Standard deviation of the measured $g$ values in 50 drops sets taken with FG5 (top) against the rms of the vertical acceleration of ground taken with the seismometer (bottom). The two instruments are located in the same laboratory and their measurements last for about 24 hours. The rms of the acceleration of ground is calculated from acquisitions taken in the same $500 \mathrm{~s}$ time interval of the FG5 acquisition sets. The plot shows correlation between the two and a lower-noise central part during the night.

Mach-Zender type, with a fixed (reference) arm and a variable (test) arm. During a drop, the motion of the test mass affects the path length of the test beam. The interference fringes that result from the recombination of the test beam and the reference beam provides an accurate measure of the motion of the test mass relative to the mass suspended on the super-spring, which provides an inertial reference frame. As the object falls, interference fringes are formed at the optical output. The interference fringes are converted to a digital signal, which is transmitted to the time interval analyzer card in the system controller. These fringes are counted and timed with an atomic clock to obtain precise time and distance pairs. A least-squares fit to these data is used to determine the value of $g$. The distance scale is given by a frequency-stabilized helium-neon laser used in the interferometer. The absolute gravity measurements are therefore directly tied to the time and length SI units.

A total of 700 time position points are recorded over the $20 \mathrm{~cm}$ length of each drop. Even if drops can be produced up to every two seconds, in routine operation, the repetition rate is $10 \mathrm{~s}$. The average of 50-100 drops is a set, which exhibits standard deviations of 40 to $150 \mathrm{~nm} / \mathrm{s}^{2}$ under normal conditions. Measurements usually consist of one or two sets per hour with the average of several sets (usually 12 to 48) providing a gravity value. The instrumental uncertainty of the FG5 is about $2 \mu \mathrm{Gal}$ as reported by the manufacturer. A software supplied by the Microg-LaCoste company is used for data acquisition. This software provides an immediate value for the local gravity, and it also includes a full-featured post-processor that allows to vary data analysis procedures and environmental corrections.

Before being used in Florence, the FG5\#238 absolute gravimeter took part in the 8th International Comparison of Absolute Gravimeters (ICAG-2009) organized by the Bureau International des Poids et Mesures (BIPM). The comparison was held from September 14th to October 3th, 2009 in Sèvres, France, and preliminary results are described in [22]. In ICAG-2009, five stations located at the BIPM were used and each gravimeter measured at three of them. The primary objective of ICAG-2009 was to determine the level of uncertainty in the absolute measurement of free-fall acceleration on the ground and to evaluate the possibility of determining a comparison reference value for $g$ at the sites of the BIPM gravity micronetwork. Such a reference value would allow correction values to be determined for the results of the gravimeters participating in the comparison. Preliminary results [22] show that the FG5\#238 absolute gravimeter measured the acceleration of gravity in three different sites with an expanded uncertainty ranging between $5.4 \mu \mathrm{Gal}$ and $6.5 \mu \mathrm{Gal}$. The expanded uncertainty is obtained by multiplying the combined standard uncertainty by a coverage factor $k$ corresponding to a confidence level of $95 \%$, in our case $k=2$. Although these results cannot be used to assure the measurement performanes of the FG5\#238 until the publication of the final report of ICAG-2009, all absolute gravity values agreed very well with the reference value of $g$ at the three different sites. For a direct comparison with the measurement uncertainties in Florence the standard uncertainty ranging between $2.7 \mu$ Gal and $3.3 \mu \mathrm{Gal}$ must be used. 

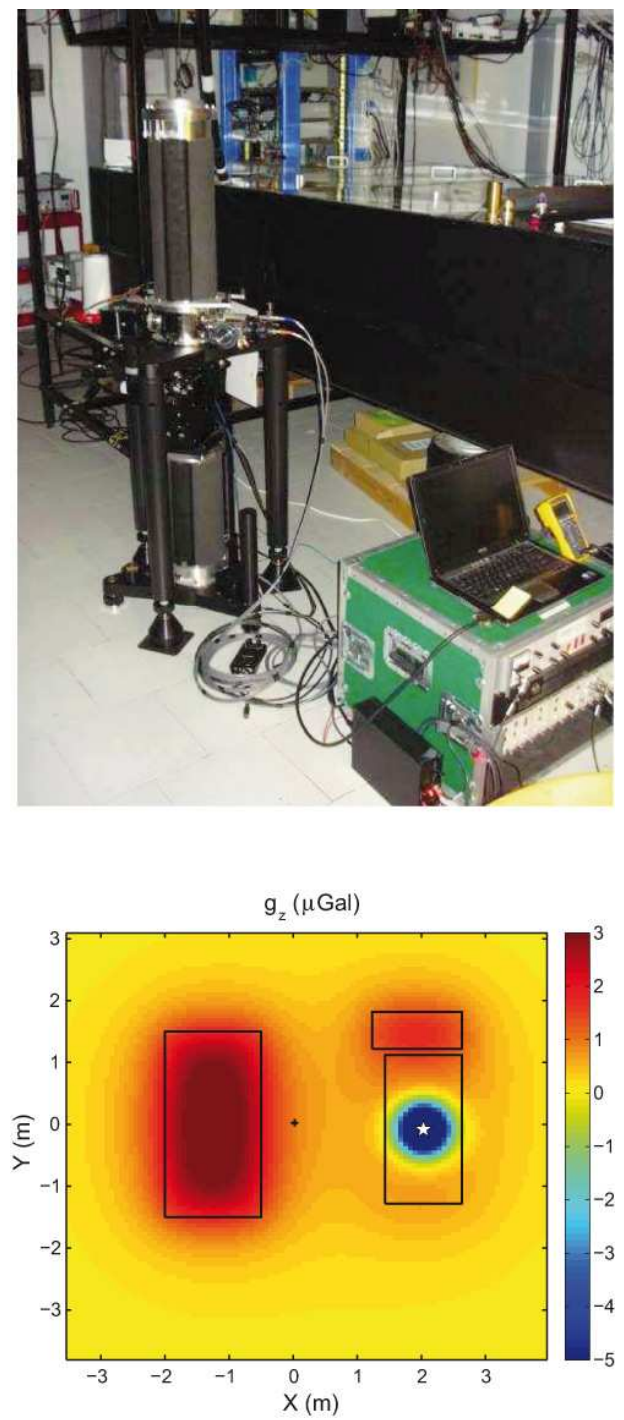

Fig. 5. (Top) View of the FG5 gravimeter installed in the atom interferometry laboratory Rb lab at the Department of Physics in Sesto Fiorentino. (Bottom) Sketch map of the laboratory with location of the masses for which we calculated the gravity field. Black rectangles are optical tables, and the star is the source mass $(\simeq 350 \mathrm{~kg}$ at the measuring time $)$ used for the determination of the Newtonian constant. The coloured map is the gravity field due to the distribution of these nearby masses at the reference height of the FG5 gravimeter $129.4 \mathrm{~cm}$ above the ground.

\subsection{Absolute gravity acceleration measurements}

After the measurements taken in ICAG-2009 the instrument has been transported to Polo Scientifico of the Florence University in Sesto Fiorentino and it has been installed first in the Sr lab at the Department of Physics and Astronomy, then in the Rb lab at LENS (see fig. 5(top)); the FG5 gravimeter was positioned in the available space as close as possible to the center of each room. The instrument was along the North-South direction within $10^{\circ}$ in order to reduce the Coriolis effect as recommended by the manufacturer. The nominal measuring point elevation for the gravimeter is $129.4 \mathrm{~cm}$ above the floor, and it coincides with the top of the drop.

In the Sr lab, acquisition started on October 4th, 2009 at about 16:30 (UTC), and lasted for about 15 hours. In the Rb lab, acquisition started on October 5th, 2009 at 10:20 (UTC), and lasted for about 24 hours. During both experiments, a measurement set every 30 minutes has been collected, each set being composed of 50 drops. Thus $[29,45]$ sets and $[1450,2250]$ launches have been collected for the two sites, respectively. On every single set the standard deviation $\sigma$ has been calculated, rejecting all the measurements resulting out of the $3 \sigma$ range. This led to 95\% accepted drops for the Sr lab, and to $99 \%$ accepted drops for the Rb lab data. All the uncertainties considered in the paper are standard uncertainties, unless it is specifically written. See, for details, table 1. 
Table 1. Summary of the relevant parameter for environmental corrections on the $g$ measurements in the Sr lab and in the Rb lab. Vertical Gravity Gradient is the free air gradient.

\begin{tabular}{|l|c|c|}
\hline & Sr lab & Rb lab \\
\hline \hline Time & $04-05 / 10 / 2009$ & $05-06 / 10 / 2009$ \\
\hline Longitude E & $11.19305^{\circ}$ & $43.19391^{\circ}$ \\
\hline Latitude N & $43.81910^{\circ}$ & $39.9 \mathrm{~m}$ \\
\hline Height a.s.l. & $40.5 \mathrm{~m}$ & $1008.46 \mathrm{mbar}$ \\
\hline Nominal Atmospheric Pressure & $1008.39 \mathrm{mbar}$ & $-0.3 \mu \mathrm{Gal} / \mathrm{mbar}$ \\
\hline Barometric factor & $-0.3 \mu \mathrm{Gal} / \mathrm{mbar}$ & $129.4 \mathrm{~cm}$ \\
\hline Reference Height & $129.4 \mathrm{~cm}$ & $3.09 \mu \mathrm{Gal} / \mathrm{cm}$ \\
\hline Vertical Gravity Gradient & $3.09 \mu \mathrm{Gal} / \mathrm{cm}$ & \\
\hline
\end{tabular}

\subsection{Data processing and correction}

Data acquisition and processing is accomplished with the software supplied by the Microg-LaCoste company, that has been also used for corrections due to systematic errors. The data are least-squares fit to a function that uses a known a priori vertical gravity gradient in a fourth-order equation of motion [21]. The finite value of the speed of light gives a correction for the calculated gravity signal since the optical interference occurs at a later time after the light is reflected by the dropped object, depending on interferometer arm length. This contribution is a non-negligible effect at the $\mu \mathrm{Gal}$ sensitivity, and the corrected delayed time has to be taken by considering a retarded time in the function used for the least-squares fit.

The vertical gravity gradient has to be taken into account because the position of the free falling mirror changes considerably $(20 \mathrm{~cm})$ along the vertical during the measurement and so does the acceleration of the falling mirror. If the drop length is $20 \mathrm{~cm}$ and the vertical gravity gradient equals the free air gradient $(3.09 \mu \mathrm{Gal} / \mathrm{cm})$, then a $1 \%$ gradient estimate error introduces a $0.2 \mu \mathrm{Gal}$ error in the measured absolute gravity value. The effect of the nearby masses (optical tables in both laboratories and source masses in the Rb lab) on the gradient has been considered on the $20 \mathrm{~cm}$ long trajectory of the FG5 falling mirror. The contribution on the gradient value is below $1 \%$ in both the $\mathrm{Sr}$ lab and the Rb lab at the FG5 sites.

Since the absolute determination of gravity with the FG5 instrument and with the atom interferometer experiments are neither carried out simultaneously nor at the same spot, gravity variations in time have to be to taken into account within each laboratory. Corrections that have been considered are Earth tides and ocean loading contributions, polar motion effects, and barometric corrections. These corrections require local instrumental measurements or geographical parameters. Moreover, to be a truly useful measurement, the sites elevations and the measured set-ups heights have been considered, and contributions due to the nearby mass distribution have been calculated.

Due to the solar and lunar attraction the local value of $g$ will change of hundreds of $\mu$ Gal. The common approach to correct observed gravity data for tidal effects is to use a tidal prediction program (such as [23]) that makes use of the observed or predicted amplitude tidal gravity effect. The surface loading of the Earth due to the weight of the ocean tides causes a time-varying deformation of the solid Earth, which is called ocean tide loading. The vertical component of the ocean tide loading varies spatially and it is in addition to the Earth's body tide deformation, which is typically $40 \mathrm{~cm}$ peak to peak in mid-latitudes.

As the Earth wobbles on its axis, the local centripetal acceleration will change the local value of $g$. This is known as Polar motion effect. The correction for polar motion has an amplitude of $\pm 10 \mu$ Gal and two principal periods of 365 days and 435 days. It is modeled with an uncertainty much better than $1 \mu \mathrm{Gal}$ [24]. By entering parameters related to the Earth's current orientation into the software, this effect can be corrected.

The barometric pressure correction has to be considered because, as the local air pressure changes, so will the measured gravity value due to direct attraction. By comparing the current pressure with the standard local value, the gravity value can be corrected to estimate the value on a "normal" day. A typical barometric factor of $-0.3 \mu \mathrm{Gal} / \mathrm{mbar}$ is considered and a contribution to the uncertainty of the gravity acceleration of $\pm 1 \mu \mathrm{Gal}$.

The manufacturer also recommends to take into account uncertainties due to laser frequency $( \pm 0.01 \mu \mathrm{Gal})$ and to Rubidium oscillator clock $( \pm 0.50 \mu \mathrm{Gal})$, together with a "set-up" uncertainty $( \pm 2 \mu \mathrm{Gal})$ depending on the instrument and on the operator.

The floor of the $\mathrm{Rb}$ lab is $61(1) \mathrm{cm}$ higher than the floor of the Sr lab. This elevation difference has been measured using a laser level Bosch mod.BL30 (nominal uncertainty $0.3 \mathrm{~mm} / \mathrm{m}$ ). The contribution of vertical gradient to the difference in the measured values of $g$ in the two locations, assuming a theoretical vertical gradient of $3.09 \mu \mathrm{Gal} / \mathrm{cm}$, 
Table 2. Summary of corrections and uncertainties on the $g$ measurements in the Sr lab and in the Rb lab. All the uncertainties are standard uncertainties and we have used as uncertainty of the result the combined standard one, in our case, the sum in quadrature of all the uncertainties. Vertical Gravity Gradient is the free air gradient and its uncertainty contribution to absoute $\mathrm{g}$ measurement is not available. The reference height of the measurements is $129.4 \mathrm{~cm}$ from the floor and heights a.s.l. are $39.9 \mathrm{~m}$ and $40.5 \mathrm{~m}$, respectively.

\begin{tabular}{|c|c|c|c|c|}
\hline & $\begin{array}{l}\text { Sr lab Value } \\
\qquad(\mu \mathrm{Gal})\end{array}$ & $\begin{array}{c}\text { Uncertainty } \\
(\mu \mathrm{Gal})\end{array}$ & $\begin{array}{l}\text { Rb lab Value } \\
\qquad(\mu \mathrm{Gal})\end{array}$ & $\begin{array}{c}\text { Uncertainty } \\
(\mu \mathrm{Gal})\end{array}$ \\
\hline Average Solid Earth Tide & 0.04 & \pm 0.09 & -88.05 & \pm 0.09 \\
\hline Average Ocean Loading Tide & -0.05 & \pm 0.10 & 0.00 & \pm 0.10 \\
\hline Measured $g$ value (tide corrected) & 980492161.7 & \pm 3.25 & 980492051.9 & \pm 1.85 \\
\hline Vertical Gravity Gradient & 3.09 & n.a. & 3.09 & n.a. \\
\hline Polar motion & -3.74 & \pm 0.05 & -3.74 & \pm 0.05 \\
\hline Barometric Pressure & 2.89 & \pm 1.00 & 1.03 & \pm 1.00 \\
\hline Set-up & 0.00 & \pm 2.00 & 0.00 & \pm 2.00 \\
\hline Laser & 0.00 & \pm 0.01 & 0.00 & \pm 0.01 \\
\hline Clock & 0.00 & \pm 0.50 & 0.00 & \pm 0.50 \\
\hline Nearby mass distribution & -0.27 & \pm 0.05 & -0.93 & \pm 0.05 \\
\hline Corrected $g$ value & 980492160.6 & \pm 4.0 & 980492048.3 & $\pm \mathbf{3 . 0}$ \\
\hline
\end{tabular}

is about $188 \mu \mathrm{Gal}$. During the measurements the rooms were occupied by optical tables and by the source masses for the Newtonian constant experiment. Their positions may change in the future, and so will their effect on gravity acceleration at the site of the FG5. Our wish is to repeat, if necessary, the FG5 measurements at the same site, that has been marked, and will be used in the future. As a consequence, we are interested in evaluating all possible signals that change with time. This is a good reason to calculate the contribution of the nearby mass distribution of the optical tables and source masses at the FG5 site for the two laboratories as shown in fig. 5. This correction is less than $1 \mu \mathrm{Gal}$ at both laboratories. An accurate gravity field description of the laboratories is beyond the aim of this paper and could be suitable if a comparison or transfer of the absolute measurement of $g$ at the level of $1 \mu \mathrm{Gal}$ is needed.

By the time of our measurements no rainfalls occurred and so we do not expect any significant gravity variations associated with changes in the local hydrological accumulation. The difference between the two values can be explained with the difference in building structures and geological structures, causes that we can assume as time invariant.

For the final value we have considered a combined standard uncertainty, in our case the sum in quadrature of all the uncertainties. After corrections due to systematics effects, the values of the acceleration of gravity in the laboratories are $(\mathbf{9 8 0} 492160.6 \pm 4.0) \mu \mathrm{Gal}$ in the Sr lab and $(\mathbf{9 8 0} 492$ 048.3 $\pm \mathbf{3 . 0}) \mu \mathrm{Gal}$ in the Rb lab. In table 2 absolute gravity acceleration values are summarized with the relevant systematic effects and the respective estimated uncertainties.

In neither the $\mathrm{Rb}$ lab nor the $\mathrm{Sr}$ lab special care has been taken in insulating the experiments from seismic noise. This is the reason for a limited uncertainty in the $g$ measurement using the atom experiments in the two laboratories. Nevertheless, as already mentioned, the comparison of the measured acceleration between the experiments and an instrument with an uncertainty of $1 \times 10^{-8}$ can be useful to check systematic effects. In the Rb lab, the atom interferometer using ultracold ${ }^{87} \mathrm{Rb}$ atoms launched in an atomic fountain is used for the precise measurement of the vertical gravity gradient and the apparatus can also be used to measure the gravitational acceleration. The acceleration of the free falling atoms is measured with Raman pulse interferometer sequence. Although the sensitivity of the atom interferometer is $\Delta g / g=5 \times 10^{-7}$, the budget of uncertainty is limited at this moment, and we derive an absolute value for the gravity acceleration $g_{R b}=980497.2 \pm 7.9 \mathrm{mGal}$.

In the $\mathrm{Sr} \mathrm{lab}$, we performed an accurate measurement of the gravitational acceleration using ultracold ${ }^{88} \mathrm{Sr}$ atoms trapped in an amplitude-modulated vertical optical lattice. When the atoms are subject only to gravity, the Bloch oscillations of an atom in a standing wave are directly related to $g$ via the atomic mass, the Planck constant, and the wavelength of the light producing the lattice. Details on the experiment and related measured $g$ are given in [6]. Tidal effects have been estimated by using the same algorithm used for the absolute gravimeter data processing. To compare the results to the FG5, we have considered sources of systematic bias like the height difference between the two instruments and the vertical alignment of the optical lattice beam. The correction for the index of refraction of the Sr cloud and the background gas in the vacuum chamber is negligible, while an important contribution to systematic shifts is due to the lattice light itself, because both the intensity and the wave vector of the lattice beam can give a 
contribution. After a set of 21 determinations of $g$ of ${ }^{88} \mathrm{Sr}$ atom we obtained $g_{S r}=980492.32 \pm 0.14 \mathrm{mGal}$, with a combined uncertainty given by the quadrature sum of the contributions coming from the fit of the amplitude modulation resonance and the uncertainties on systematic corrections. In conclusion, we have obtained a good agreement with the values measured with the FG5 gravimeter in the same locations, although the uncertainty of atom sensors is limited to a level of $10^{-7}$ for the strontium experiment and to $10^{-6}$ for the rubidium experiment.

\section{Conclusions}

The measurement of the absolute acceleration of gravity $g$ has been carried out in two laboratories at Polo Scientifico of Florence University in Sesto Fiorentino: the Rb lab (Department of Physics), where atom interferometry is used for accurate measurement of the Newtonian constant, and the Sr lab (LENS), where a cold strontium atom sample is used for precision gravity measurements and for a test at short distances of the Newtonian law.

A geological description of the site is reported and in order to quantify the noise conditions at the measuring site, we have conducted a microseismic survey during an 8 day time covering the time interval of the gravimetric measurements. The seismic noise spectra show that ground vibration at day time are 2.5-3 times larger than those observed during the night, due to the intense human activities conducted both inside and in proximity of the laboratories.

We have taken into account gravity variations in time and in space within each laboratory. Gravity corrections considered are Earth tides and ocean loading contributions, polar motion and barometric effects, site elevation, measured set-up and height corrections. We have also introduced other corrections due to the nearest mass distribution near the FG5 location. During the measurements procedures the FG5\#238 absolute gravimeter worked reliably, and the uncertainties of the absolute gravity acceleration in the $\mathrm{Sr}$ and $\mathrm{Rb}$ laboratories are $4.0 \mu \mathrm{Gal}$ and $3.0 \mu \mathrm{Gal}$, respectively. A comparison between the atom acceleration values and the FG5 results has been realised, that can check our instrument against various systematic effects. However, since there is no guarantee that the gravity acceleration value is constant at these two sites, repeated measurements in time might be especially important to verify the stability of the sites.

The authors acknowledge financial support from INFN and from CNR under ESF-Eurocores EUROQuasar-IQS project. The authors wish to thank ENI S.p.a., Exploration \& Production Division for providing the FG5\#238 absolute gravimeter. COREMO, the seismological instruments facility at the INGV — Centro Nazionale Terremoti - is greatly acknowledged for having provided both seismometers and portable recorders. MdA also thanks Lorenzo Arcidiaco from LAMMA for the site map from Regione Toscana.

\section{References}

1. M. de Angelis, A. Bertoldi, L. Cacciapuoti, A. Giorgini, G. Lamporesi, M. Prevedelli, G. Saccorotti, F. Sorrentino, G.M. Tino, Meas. Sci. Technol. 20, 022001 (2009).

2. M. Fattori, G. Lamporesi, T. Petelski, J. Stuhler, G.M. Tino, Phys. Lett. A 318, 184 (2003).

3. G. Lamporesi, A. Bertoldi, L. Cacciapuoti, M. Prevedelli, G.M. Tino, Phys. Rev. Lett. 100, 050801 (2008).

4. F. Sorrentino, L. Cacciapuoti, Y.-H. Lien, M. Prevedelli, G. Rosi, G.M. Tino, New J. Phys. 12, 095009 (2010).

5. F. Sorrentino, A. Alberti, G. Ferrari, V.V. Ivanov, N. Poli, M. Schioppo, G.M. Tino, Phys. Rev. A 79, 13409 (2009).

6. N. Poli, F.-Y. Wang, M.G. Tarallo, A. Alberti, M. Prevedelli, G.M. Tino, Phys. Rev. Lett. 106, 038501 (2011).

7. H. Müller, A. Peters, S. Chu, Nature 463, 926 (2010).

8. I. Marson, F. Palmieri, Boll. Geod. Sci. Affini - IGM LIII, 161 (1994).

9. G. D'Agostino, S. Desogus, A. Germak, C. Origlia, D. Quagliotti, G. Berrino, G. Corrado, V. D'Errico, G. Ricciardi, Ann. Geophys. 51, 39 (2008).

10. Alessandro Germak, INRIM Torino, private communication.

11. A. Peters, K.Y. Chung, S. Chu, Nature 400, 849 (1999).

12. G. Ferrari, N. Poli, F. Sorrentino, G.M. Tino, Phys. Rev. Lett. 97, 060402 (2006).

13. V.V. Ivanov, A. Alberti, M. Schioppo, G. Ferrari, M. Artoni, M.L. Chiofalo, G.M. Tino, Phys. Rev. Lett. 100, 043602 (2008).

14. M. Boccaletti, G. Corti, P. Gasperini, L. Piccardi, G. Vannucci, S. Clemente, Pure Appl. Geophys. 158, 2313 (2001).

15. F. Capecchi, G. Guazzone, G. Pranzini, Boll. Soc. Geol. It. 94, 637 (1975).

16. T. Crespellani, A. Ghinelli, C. Madiai, G. Vannucchi, Stratigraphical profiles and geotechnical properties, in Seismic Hazard and Site Effects in the Florence Area, Proceedings of the 10th Eur. Conf. Geot. Eng. (ECSMFE), Florence, May 1991, Vol. IV (1991) pp. 1491-1508.

17. D.E. McNamara, R.P. Buland, Bull. Seism. Soc. Am. 94, 1517 (2004).

18. P. Welch, IBM J. Res. Dev. 5, 141 (1967).

19. J. Peterson, Observations and Modeling of Seismic Background Noise, USGS Open file Report (1993) pp. 93-322. 
20. A. Peters, K.Y. Chung, S. Chu, Metrologia 38, 25 (2001).

21. T.M. Niebauer, G.S. Sagasawa, J.E. Faller, R. Hilt, F. Klopping, Metrologia 32, 159 (1995).

22. E.F. Arias, Z. Jiang, L. Robertsson, L.F. Vitushkin, The first M.G-K1 Key Comparison: International Comparison of Absolute Gravimeters ICAG2009, in Proceedings of the IAG Symposium on Terrestrial Gravimetry: Static and Mobile Measurements (TG-SMM2010) 22-25 June, Saint Petersburg, edited by D.O. Taranovskiy (2011) p. 64.

23. G. Wenzel, ETGTAB: Earth tide prediction program (2002).

24. J.M. Wahr, J. Geophys. Res. 90, 9363 (1985). 